\title{
The role of serum D-Dimer for the diagnosis of periprosthetic shoulder infection
}

\author{
Doruk Akgün $^{1}$ · Faisal Al-Muhtaresh ${ }^{1}$ - Alp Paksoy ${ }^{1}$. Lucca Lacheta ${ }^{1} \cdot$ Marvin Minkus $^{1}$ - Daniel Karczewski ${ }^{1}$. \\ Philipp Moroder ${ }^{1}$
}

Received: 18 August 2021 / Accepted: 5 February 2022 / Published online: 19 February 2022

(c) The Author(s) 2022

\begin{abstract}
Introduction D-Dimer was recently identified as an additional biomarker in the diagnosis of hip and knee periprosthetic joint infection (PJI). Currently, there is only one study in literature dealing with the role of D-Dimer in the diagnosis of shoulder PJI. The purpose of this study was, therefore, to validate the sensitivity and specificity of D-Dimer in detecting shoulder PJI. Materials and methods All patients, who underwent septic or aseptic revision shoulder arthroplasty in our institution between November 2018 und March 2021, were analyzed. Our cohort consisted of 30 patients, of that 14 (47\%) had a shoulder PJI according the last proposed criteria of the International Consensus Meeting. The diagnostic validity of serum D-Dimer regarding the detection of PJI was analyzed.

Results The mean D-Dimer level was significantly higher for the patients with shoulder PJI compared to patients with aseptic failure $(1.44 \pm 1 \mathrm{mg} / \mathrm{l}$ vs. $0.76 \pm 0.6 \mathrm{mg} / \mathrm{l}, p=0.025)$. Coagulase-negative staphylococci were the most commonly isolated pathogens, in $9 / 14$ patients (64\%), followed by Cutibacterium acnes in 5/14 patients (36\%). According to the ROC analysis, a serum D-Dimer threshold of $0.75 \mathrm{mg} / \mathrm{l}$ had a sensitivity of $86 \%$ and a specificity of $56 \%$ for detection of a shoulder PJI. The area under curve was 0.74 . A serum C-reactive protein (CRP) cutoff of $10 \mathrm{mg} / \mathrm{l}$ showed a sensitivity of $69 \%$ and a specificity of $88 \%$. When both serum D-Dimer and CRP above the thresholds of $0.75 \mathrm{mg} / \mathrm{l}$ and $10 \mathrm{mg} / \mathrm{l}$, respectively, were used to identify a PJI the sensitivity and specificity were $57 \%$ and $100 \%$, respectively.

Conclusions Serum D-Dimer showed a good sensitivity but a poor specificity for the diagnosis of shoulder PJI. Combination D-Dimer and CRP led to improvement of the specificity, however, at the cost of sensitivity. Thus, combination of both methods may be used as a confirmatory test in the diagnosis of shoulder PJI but not to rule out infection.
\end{abstract}

Level of evidence Diagnostic level II.

Keywords Periprosthetic shoulder infection $\cdot$ D-Dimer $\cdot$ Validity $\cdot$ Diagnostics

\section{Introduction}

Periprosthetic joint infection (PJI) of the shoulder occurs in around $1 \%$ of the cases after primary and up to $15 \%$ after revision arthroplasty $[1,2]$ and the absolute number of patients with shoulder PJI is expected to rise with the

Doruk Akgün and Faisal Al-Muhtaresh equally contributed to this work.

Doruk Akgün

doruk.akguen@charite.de

1 Center for Musculoskeletal Surgery, Berlin Institute of Health, Charité - Universitätsmedizin Berlin, Freie Universität Berlin, Humboldt-Universität Zu Berlin, Augustenburger Platz 1, 13353 Berlin, Germany increasing number of performed shoulder arthroplasties [3]. The ensuing individual as well as social and economic burden is substantial [1]. The timely and correct diagnosis of shoulder PJI holds the key to a successful treatment of these patients with a lasting infection-free survival. Despite the increase of research dealing with the diagnosis of PJI in recent years, shoulder PJI remains a diagnostic challenge due to the ambiguity presented by its unique microbiologic profile with low-virulent microorganisms and stealth-type clinical appearance $[4,5]$. Widely used biomarkers in the diagnosis of PJI, such as C-reactive protein (CRP) or erythrocyte sedimentation rate (ESR) have a very limited value in the detection of low-grade infections misdiagnosing more than one third of the patients [6-9]. Therefore, serological 
tests that can help to diagnose PJI with a higher accuracy are of interest.

Recently, D-Dimer was described as a promising marker for the diagnosis of hip and knee PJI by Shahi et al. [10] and declared as a minor criterium in the consensus definition of knee and hip PJI [11]. However, there is scarce and conflicting evidence supporting its use for the diagnosis of PJI in recent literature, which shows a very limited diagnostic value compared with traditional biomarkers (ESR and CRP) [12-14]. Although the number of studies reporting on the role of D-Dimer in the diagnosis of hip and knee PJI are increasing, currently there is only one study in literature dealing with the role of D-Dimer in the diagnosis of shoulder PJI, showing a limited diagnostic utility with a sensitivity and specificity of $61 \%$ and $74 \%$, respectively [15]. The purpose of this study was, therefore, to validate the sensitivity and specificity of D-Dimer in detecting shoulder PJI.

\section{Materials and methods}

\section{Study design and cohort}

This was a cross-sectional study with analysis of collected data of 32 patients, who underwent septic or aseptic revision shoulder arthroplasty in our institution between November 2018 und March 2021. Exclusion criteria were recent history of trauma or previous surgery, as well as history of venous thrombosis, other ongoing infections or coagulation disorder including malignancy or autoimmune diseases. Excluded were two patients with a history of trauma (within 2 weeks) and with a previous surgery within 2 weeks prior to revision surgery, so our cohort consisted of 30 patients. The study protocol was reviewed and approved by the institutional ethics committee (EA4/040/14).

Following data was recorded prospectively for each patient: gender and age, involved joint, clinical symptoms, surgical history of the involved joint, type of arthroplasty, time interval between primary arthroplasty and revision surgery, concurrent antibiotic treatment, as well as radiological assessment.

\section{Standard diagnostic protocol and definition of shoulder PJI}

The standard diagnostic protocol to identify PJI included the following assessments in all patients; Laboratory values including C-reactive protein (CRP), serum D-Dimer, serum leucocyte count as well as results of preoperative aspiration, if performed, including leucocyte count, neutrophil percentage, microbiologic and histopathologic results. Furthermore, radiological and intraoperative evaluation of the component loosening and intraoperative findings, such as cloudy fluid or gross intra-articular purulence were performed and documented.

Preoperative aspiration was performed routinely in every case and was successful in 17 patients (57\%). According to the standard PJI protocol at our institution at least 5 periprosthetic tissue cultures from various suspicious surgical sites, at least one specimen for histopathologic analysis and retrieved implants for sonication analysis were obtained in every patient at the time of revision surgery. Specimen for microbiological analysis were collected with a new sterile instrument each time, were placed directly into sterile containers without touching by hand and sent immediately with retrieved implants to our microbiology laboratory for further analysis within $1 \mathrm{~h}$ after surgery. The microbiologic specimen as well as sonication fluid cultures are plated onto aerobic and anaerobic sheep blood agar plates and incubated for 14 days. Sonication was performed as previously described [16]. Shoulder PJI was diagnosed according to the last proposed definition criteria of the ICM [17].

\section{Statistical analysis}

Chi-squared and Fisher's exact tests were used to find significant differences between categorical variables. The twosample $t$ test (for parametric distribution) or Mann-Whitney $U$ test (for non-parametric distribution) was used to compare continuous variables between groups. For analysis of the diagnostic utility of serum D-Dimer, patients in the definitive, probable and possible infection groups were combined and defined as infection group and patients in infection unlikely group were defined as non-infection group. Sonication fluid cultures were incorporated into the infection criteria as microbiologic results used for the infection definition. Receivers operating characteristic (ROC) analysis was performed to display sensitivity and specificity of serum D-Dimer level for shoulder PJI. The area under the curve was calculated, and the optimum cutoff point was determined by the maximized Youden`s index. The results were expressed as mean and standard deviation (SD) or as number and percentage. A $p$ value $<0.05$ was considered significant. SPSS version 20 (SPSS Inc., Chicago, Illinois) was used for the statistical analyses.

\section{Results}

The mean age and standard deviation (SD) of the patients were $69.1 \pm 10.4$ years and 18 patients were females $(60 \%)$. Ten patients $(33 \%)$ had a previous revision surgery, three patients due to a septic reason. The mean interval between the primary arthroplasty and revision surgery were $4.9 \pm 6.2$ years. The type of arthroplasty at the time of revision surgery was hemiarthroplasty in nine patients, 
total shoulder arthroplasty in three and reverse shoulder arthroplasty in 18 patients. None of the patients had a history of venous thrombosis or coagulation disorder including malignancy or autoimmune diseases. The reasons for revision surgery included beside infection, loosening of the components, overstuffing, secondary rotator cuff insufficiency and instability.

A total of 14 patients were $(47 \%)$ were identified as infected, 6 meeting the criteria of definitive infection, 6 of probable infection and 2 of possible infection. The mean D-Dimer level was significantly higher for the patients with shoulder PJI compared to patients with aseptic failure $(1.44 \pm 1 \mathrm{mg} / \mathrm{l}$ vs. $0.76 \pm 0.6 \mathrm{mg} / \mathrm{l}, p=0.025)$, as well as the mean CRP level $(26 \pm 30 \mathrm{mg} / \mathrm{l}$ vs. $4.2 \pm 4 \mathrm{mg} / \mathrm{l}, p=0.009)$. A detailed demographic and clinical comparison of both groups is demonstrated in Table 1.

In all but two of infected patients at least one microorganism could have been identified and in 5 patients a polymicrobial infection was evident. Coagulase-negative staphylococci were the most commonly isolated pathogens, in $9 / 14$ patients (64\%), followed by Cutibacterium acnes in 5/14 patients (36\%), Bacillus cereus in two patients and Staphylococcus aureus and Streptococcus parasanguinus each in one patient. The mean time to revision from the primary arthroplasty was longer for the infection group than non-infection group, however, statistically not significant $(6.3 \pm 8$ years vs. $3.6 \pm 5$ years, $p=0.3)$. According to the ROC analysis, a serum D-Dimer threshold of $0.75 \mathrm{mg} / \mathrm{l}$ had a sensitivity of $86 \%$ and a specificity of $56 \%$, a positive predictive value (PPV) of $63 \%$, a negative predictive value (NPV) of $82 \%$ and an accuracy of $70 \%$ for detection of a shoulder PJI. The area under curve
(AUC) was 0.74 and the ROC analysis had a significance level of 0.026. Table 2 shows different thresholds with sensitivity and specificity. A serum CRP cutoff of $10 \mathrm{mg} / \mathrm{l}$ as recommended by ICM [17], showed a sensitivity of $69 \%$ and a specificity of $88 \%$, a PPV of $82 \%$, a NPV of $79 \%$ and an accuracy of $80 \%$. When both serum D-Dimer and CRP above the thresholds of $0.75 \mathrm{mg} / \mathrm{l}$ and $10 \mathrm{mg} / \mathrm{l}$, respectively, were used to identify a positive result the sensitivity, specificity, PPV, NPV and accuracy were $57 \%$, $100 \%, 100 \%, 73 \%$ and $80 \%$, respectively.

Table 2 Sensitivities and specificities for different D-Dimer thresholds

\begin{tabular}{lcc}
\hline $\begin{array}{l}\text { D-Dimer threshold } \\
(\mathrm{mg} / \mathrm{l})\end{array}$ & Sensitivity (\%) & Specificity (\%) \\
\hline 0.24 & 100 & 6 \\
0.31 & 100 & 19 \\
0.4 & 100 & 44 \\
0.66 & 86 & 44 \\
0.75 & 86 & 56 \\
0.85 & 71 & 62 \\
0.9 & 64 & 62 \\
1 & 43 & 75 \\
1.7 & 36 & 94 \\
2.5 & 14 & 94 \\
3.1 & 7 & 100 \\
\hline
\end{tabular}

Table 1 Patient demographic and clinical characteristics

\begin{tabular}{lllll}
\hline Variable & $\begin{array}{l}\text { Infection } \\
\text { group, } n=14\end{array}$ & $\begin{array}{l}\text { Non-infection } \\
\text { group, } n=16\end{array}$ & All patients, $n=30$ & $p$ value $^{\mathrm{a}}$ \\
\hline $\begin{array}{l}\text { Age at revision surgery (year)* } \\
\text { Sex }\end{array}$ & $72.4 \pm 10$ & $66.2 \pm 10$ & $69.1 \pm 10$ & 0.1 \\
Male & $5(36)$ & $7(44)$ & $12(40)$ & 0.7 \\
Female & $9(62)$ & $9(56)$ & $18(60)$ & \\
Type of arthroplasty & & & \\
Hemi & $3(21)$ & $6(38)$ & $9(30)$ & \\
TSA & $3(21)$ & $0(0)$ & $3(10)$ & \\
RSA & $8(57)$ & $10(62)$ & $18(60)$ \\
Interval between index and revi- & $6.3 \pm 8$ & $3.6 \pm 5$ & $4.9 \pm 6.2$ & 0.3 \\
sion arthroplasty (year)* & & & \\
CRP at admission (mg/l)* & $26 \pm 30$ & $4.2 \pm 4$ & $14.3 \pm 23$ \\
D-Dimer at admission (mg/l)* & $1.4 \pm 1$ & $0.76 \pm 0.6$ & $1.1 \pm 0.9$ & 0.009 \\
\hline
\end{tabular}

TSA Total shoulder arthroplasty, RSA Reverse shoulder arthroplasty, CRP C-reactive protein

*The values are given as the mean and the standard deviation

The values are given as the number with the percentage of the group in parentheses

${ }^{\mathrm{a}}$ The statistical analysis was only done between infection and non-infection groups 


\section{Discussion}

Shoulder PJI presents a unique diagnostic challenge because of the low virulence of the most common causative microorganisms. The determination of the infection status in patients with a clinically failed shoulder arthroplasty is a key step in the treatment planning. Therefore, the identification of potential biomarkers for an accurate diagnosis of shoulder PJI is of importance. Recent research focused on the role of D-Dimer in the diagnosis of knee and hip PJI with conflicting results. To our knowledge there is only one study evaluating the role of serum D-Dimer in the diagnosis of shoulder PJI. Our results showed a lower accuracy of D-Dimer compared to CRP in the diagnosis of shoulder PJI, D-Dimer with a higher sensitivity and CRP with a higher specificity. Combination of both methods led to improvement of the specificity, however, at the cost of sensitivity. Thus, combination of both methods may be used as a confirmatory test in the diagnosis of shoulder PJI.

D-Dimer is mostly used as a screening tool for deep vein thrombosis or pulmonary embolism in lower limbs [18]. Recent literature showed an increase of D-Dimer in the setting of systemic inflammation and infection, especially in a joint $[19,20]$. Rodelo et al. reported on the prognostic role of D-Dimer in patients with sepsis showing that high level of D-Dimer was associated with an increased 28-day mortality [21]. Shahi et al. first reported on the role of D-Dimer in the diagnosis of hip and knee PJI in a prospective study [10]. They showed a sensitivity and specificity of $89 \%$ and $93 \%$, respectively, for a determined optimal threshold value of $0.85 \mathrm{mg} / \mathrm{l}$, which is similar to the value identified in our study by the Youden`s index. Furthermore, they concluded that D-Dimer was also accurate in predicting the presence of infection at the time of reimplantation during a two-stage exchange arthroplasty. In another study Quin et al. identified the D-Dimer as a valuable biomarker in detecting chronic hip and knee PJI with a sensitivity and specificity of $93 \%$ and $75 \%$, respectively, with an optimal threshold of $1.170 \mathrm{mg} / \mathrm{l}$. In contrast to our study the authors showed, that a combination of D-Dimer with CRP led to improvement of sensitivity (98\%), however, at the cost of specificity (42\%). Thus, they recommended use of the negativity of serum D-Dimer and CRP to rule out a PJI [22]. Contrary to these findings, Hong Xu et al. reported a lower sensitivity and specificity of serum D-Dimer (69\% and 51\%, respectively) compared to CRP, ESR and interleukin-6 with a threshold of $1.02 \mathrm{mg} / 1$ [12]. Thus, they stated that D-Dimer has a limited value for diagnosing hip and knee PJI. Similar to these results, Rui li et al. found a lower sensitivity and specificity of plasma
D-Dimer (64\% and 65\%, respectively) in the diagnosis of knee and hip PJI compared to CRP and ESR. They also analyzed plasma fibrinogen, another coagulation-related indicator, and showed a promising performance similar with CRP and ESR. In another study of Pannu et al. serum D-Dimer showed a poor accuracy to discriminate between septic and aseptic cases in the setting of hip and knee arthroplasty revision [14]. Zmistowski et al. were recently able to show a limited diagnostic utility of serum D-Dimer similar to other serum biomarker in identifying patients with a periprosthetic shoulder infection [15]. In their study, a serum D-Dimer threshold of $0.6 \mathrm{mg} / \mathrm{l}$ showed a sensitivity and specificity of $61 \%$ and $74 \%$, respectively.

In contrast to the results of Zmistowski et al. our study showed a good sensitivity but a poor specificity of D-Dimer in the diagnosis of shoulder PJI using a threshold of $0.75 \mathrm{mg} / \mathrm{l}$. As mentioned above, the recommended threshold differs in recent studies and a universal threshold for D-Dimer in diagnosing PJI still remains unknown. Moreover, the inflammatory response of shoulder PJI may be much more different from that of hip and knee PJI due to the majority of low-virulence microorganisms, effecting the optimal serum D-Dimer threshold. In addition, in our study, only two patients were infected with a highvirulent microorganism. As presented in recent studies, less virulent PJIs had been associated with low CRP values $[6,7,9]$. The hypothesis that the CRP response in patients with a PJI caused by low-virulent microorganisms may be week, may be also true for serum D-Dimer. Therefore, the detection of low-grade shoulder PJIs may be very challenging despite combination of both methods. However, combination of both methods may be used as a confirmatory test in the diagnosis of shoulder PJI meaning that the combination of an elevated D-Dimer and CRP serum level in an otherwise healthy patient with symptomatic shoulder arthroplasty indicates PJI with a high likelihood.

This study has some limitations. The small size of the study cohort can alter our results. However, the ROC analysis had a significance level of 0.026 , which shows enough power of our sample size. While we don't believe, that including more patients would change the results significantly, the conclusions drawn from this rather small cohort should be confirmed in future studies. Despite none of the patients in our cohort had a history of venous thrombosis or coagulation disorder including malignancy or autoimmune diseases, this was only based on the patient's statements. The possibly raised D-Dimer levels in patients with venous thrombosis or coagulation disorder may alter our results and decrease the specificity of combination of CRP and D-Dimer. In these patients both methods may not be used as a confirmatory test. 


\section{Conclusion}

Serum D-Dimer showed a good sensitivity but a poor specificity for the diagnosis of shoulder PJI. Combination of D-Dimer and CRP led to improvement of the specificity, however, at the cost of sensitivity. Thus, combination of both methods may be used as a confirmatory test in the diagnosis of shoulder PJI but not to rule out infection.

Funding Open Access funding enabled and organized by Projekt DEAL. No funding was received for this research.

Availability of data and materials Not applicable.

Code availability Not applicable.

\section{Declarations}

Conflict of interest The authors declare that they have no conflict of interest.

Ethical approval The study protocol (Application number: EA4/040/14) was reviewed and approved by the institutional review board (Charite Universitätsmedizin).

Consent to participate Not applicable.

Consent for publication Not applicable.

Open Access This article is licensed under a Creative Commons Attribution 4.0 International License, which permits use, sharing, adaptation, distribution and reproduction in any medium or format, as long as you give appropriate credit to the original author(s) and the source, provide a link to the Creative Commons licence, and indicate if changes were made. The images or other third party material in this article are included in the article's Creative Commons licence, unless indicated otherwise in a credit line to the material. If material is not included in the article's Creative Commons licence and your intended use is not permitted by statutory regulation or exceeds the permitted use, you will need to obtain permission directly from the copyright holder. To view a copy of this licence, visit http://creativecommons.org/licenses/by/4.0/.

\section{References}

1. Padegimas EM, Maltenfort M, Ramsey ML, Williams GR, Parvizi J, Namdari S (2015) Periprosthetic shoulder infection in the US: incidence and economic burden. J Shoulder Elbow Surg 24:741-746. https://doi.org/10.1016/j.jse.2014.11.044

2. Singh JA, Sperling JW, Schleck C, Harmsen WS, Cofield RH (2012) Periprosthetic infections after total shoulder arthroplasty: a 33-year perspective. J Shoulder Elbow Surg 21:15341541. https://doi.org/10.1016/j.jse.2012.01.006

3. Paxton ES, Green A, Krueger VS (2019) Periprosthetic infections of the shoulder: diagnosis and management. J Am Acad Orthop Surg 27:e935-e944. https://doi.org/10.5435/ JAAOS-D-18-00232
4. Hsu JE, Somerson JS, Vo KV, Matsen FA 3rd (2017) What is a "periprosthetic shoulder infection"? A systematic review of two decades of publications. Int Orthop 41:813-822. https://doi.org/ 10.1007/s00264-017-3421-6

5. Akgun D, Maziak N, Plachel F, Siegert P, Minkus M, Thiele K, Moroder P (2020) The role of implant sonication in the diagnosis of periprosthetic shoulder infection. J Shoulder Elbow Surg. https://doi.org/10.1016/j.jse.2019.10.011

6. Akgun D, Burger J, Pumberger M, Putzier M (2019) C-reactive protein misdiagnoses delayed postoperative spinal implant infections in patients with low-virulent microorganisms. Eur Spine J. https://doi.org/10.1007/s00586-019-05889-3

7. Akgun D, Muller M, Perka C, Winkler T (2018) The serum level of C-reactive protein alone cannot be used for the diagnosis of prosthetic joint infections, especially in those caused by organisms of low virulence. Bone Jt J 100-B:1482-1486. https://doi. org/10.1302/0301-620X.100B11.BJJ-2018-0514.R1

8. Piper KE, Fernandez-Sampedro M, Steckelberg KE, Mandrekar JN, Karau MJ, Steckelberg JM, Berbari EF, Osmon DR, Hanssen AD, Lewallen DG, Cofield RH, Sperling JW, Sanchez-Sotelo J, Huddleston PM, Dekutoski MB, Yaszemski M, Currier B, Patel R (2010) C-reactive protein, erythrocyte sedimentation rate and orthopedic implant infection. PLoS ONE 5:e9358. https://doi.org/ 10.1371/journal.pone.0009358

9. Akgun D, Wietholter M, Siegert P, Danzinger V, Minkus M, Braun KF, Moroder P (2021) The role of serum C-reactive protein in the diagnosis of periprosthetic shoulder infection. Arch Orthop Trauma Surg. https://doi.org/10.1007/s00402-021-03779-2

10. Shahi A, Kheir MM, Tarabichi M, Hosseinzadeh HRS, Tan TL, Parvizi J (2017) Serum D-Dimer test is promising for the diagnosis of periprosthetic joint infection and timing of reimplantation. $\mathrm{J}$ Bone Joint Surg Am 99:1419-1427. https://doi.org/10.2106/JBJS. 16.01395

11. Shohat N, Bauer T, Buttaro M, Budhiparama N, Cashman J, Della Valle CJ, Drago L, Gehrke T, Marcelino Gomes LS, Goswami K, Hailer NP, Han SB, Higuera CA, Inaba Y, Jenny JY, KjaersgaardAndersen P, Lee M, Llinas A, Malizos K, Mont MA, Jones RM, Parvizi J, Peel T, Rivero-Boschert S, Segreti J, Soriano A, Sousa R, Spangehl M, Tan TL, Tikhilov R, Tuncay I, Winkler H, Witso E, Wouthuyzen-Bakker M, Young S, Zhang X, Zhou Y, Zimmerli W (2019) Hip and knee section, what is the definition of a periprosthetic joint infection (PJI) of the knee and the hip? Can the same criteria be used for both joints?: proceedings of international consensus on orthopedic infections. J Arthroplasty 34:S325-S327. https://doi.org/10.1016/j.arth.2018.09.045

12. Xu H, Xie J, Huang Q, Lei Y, Zhang S, Pei F (2019) Plasma fibrin degradation product and $\mathrm{D}$-dimer are of limited value for diagnosing periprosthetic joint infection. J Arthroplasty 34:2454-2460. https://doi.org/10.1016/j.arth.2019.05.009

13. Li R, Shao HY, Hao LB, Yu BZ, Qu PF, Zhou YX, Chen JY (2019) Plasma fibrinogen exhibits better performance than plasma D-dimer in the diagnosis of periprosthetic joint infection: a multicenter retrospective study. J Bone Joint Surg Am 101:613-619. https://doi.org/10.2106/JBJS.18.00624

14. Pannu TS, Villa JM, Patel PD, Riesgo AM, Barsoum WK, Higuera CA (2020) The utility of serum d-dimer for the diagnosis of periprosthetic joint infection in revision total hip and knee arthroplasty. J Arthroplasty. https://doi.org/10.1016/j.arth.2020. 01.034

15. Zmistowski B, Chang M, Shahi A, Nicholson T, Abboud J, Lazarus M, Williams G, Parvizi J, Namdari S (2021) Is D-dimer a reliable serum marker for shoulder periprosthetic joint infection? Clin Orthop Relat Res. https://doi.org/10.1097/CORR.00000 00000001774

16. Renz N, Feihl S, Cabric S, Trampuz A (2017) Performance of automated multiplex PCR using sonication fluid for diagnosis 
of periprosthetic joint infection: a prospective cohort. Infection 45:877-884. https://doi.org/10.1007/s15010-017-1073-5

17. Garrigues GE, Zmistowski B, Cooper AM, Green A, Group ICMS (2019) Proceedings from the 2018 international consensus meeting on orthopedic infections: the definition of periprosthetic shoulder infection. J Shoulder Elbow Surg 28:S8-S12. https://doi. org/10.1016/j.jse.2019.04.034

18. Chen CJ, Wang CJ, Huang CC (2008) The value of D-dimer in the detection of early deep-vein thrombosis after total knee arthroplasty in Asian patients: a cohort study. Thromb J 6:5. https://doi. org/10.1186/1477-9560-6-5

19. Ribera T, Monreal L, Armengou L, Rios J, Prades M (2011) Synovial fluid D-dimer concentration in foals with septic joint disease. J Vet Intern Med 25:1113-1117. https://doi.org/10.1111/j.19391676.2011.0758.x

20. Schwameis M, Steiner MM, Schoergenhofer C, Lagler H, Buchtele N, Jilma-Stohlawetz P, Boehm T, Jilma B (2015) D-dimer and histamine in early stage bacteremia: a prospective controlled cohort study. Eur J Intern Med 26:782-786. https://doi.org/10.1016/j. ejim.2015.10.024

21. Rodelo JR, De la Rosa G, Valencia ML, Ospina S, Arango CM, Gomez CI, Garcia A, Nunez E, Jaimes FA (2012) D-dimer is a significant prognostic factor in patients with suspected infection and sepsis. Am J Emerg Med 30:1991-1999. https://doi.org/10. 1016/j.ajem.2012.04.033

22. Qin L, Li F, Gong X, Wang J, Huang W, Hu N (2020) Combined measurement of D-Dimer and C-reactive protein levels: highly accurate for diagnosing chronic periprosthetic joint infection. J Arthroplasty 35:229-234. https://doi.org/10.1016/j.arth.2019.08. 012

Publisher's Note Springer Nature remains neutral with regard to jurisdictional claims in published maps and institutional affiliations. 